\title{
Contribution of PET/CT to Prediction of Outcome in Children and Young Adults with Rhabdomyosarcoma
}

\author{
Sven H. Baum ${ }^{1}$, Michael Frühwald ${ }^{2}$, Kambiz Rahbar ${ }^{1}$, Johannes Wessling ${ }^{3}$, Otmar Schober ${ }^{1}$, and Matthias Weckesser ${ }^{1}$ \\ ${ }^{1}$ Department of Nuclear Medicine, Münster University Hospital, Münster, Germany; ${ }^{2}$ Department of Pediatric Hematology and \\ Oncology, Münster University Hospital, Münster, Germany; and ${ }^{3}$ Department of Clinical Radiology, Münster University Hospital, \\ Münster, Germany
}

The purpose of this retrospective study was to evaluate the role of ${ }^{18} \mathrm{~F}-\mathrm{FDG}$ PET or PET/CT in the prediction of patient outcome in children and young adults affected by rhabdomyosarcoma. Methods: Forty-one patients with histology-proven rhabdomyosarcoma who underwent PET or PET/CT were identified (age range, 1-20 y; mean age $\pm \mathrm{SD}, 9.9 \pm 5.8$ y). Tumor maximum standardized uptake value $\left(S U V_{\max }\right)$ and visually rated metabolic activity, as well as the presence of metabolically active lymph nodes and distant metastases, were compared with event-free and overall survival. Multivariate Cox regression analyses were performed to compare the prediction of outcome according to metabolic tumor intensity in relation to established prognostic factors. Results: Kaplan-Meier analyses revealed a significantly shorter overall survival in primary tumors visually rated as highly metabolically active or with a ratio of SUV $V_{\max }$ to SUV of the liver above 4.6. In addition, metabolically active lymph node and distant site involvement was indicative of significantly lower survival rates. On multivariate Cox regression analysis, the impact of intensity or SUV $\mathrm{max}_{\max }$ of the primary tumor on outcome failed to attain significance, although PET performed better than some of the prognostic factors established in larger patient groups $(P=0.081)$. Conclusion: ${ }^{18} \mathrm{~F}-\mathrm{FDG} \mathrm{PET} /$ CT is a valuable tool for initial staging in children affected by rhabdomyosarcoma. ${ }^{18} \mathrm{~F}-\mathrm{FDG}$ PET/CT may be an additional predictor of outcome and may be used to refine risk-adapted therapy. PET performed better than some established risk factors. The borderline significance level of primary tumor metabolism in multivariate testing may be an effect of the limited sample size. Further prospective evaluations are warranted.

Key Words: ${ }^{18}$ F-FDG PET/CT; rhabdomyosarcoma; prognosis; risk stratification

J Nucl Med 2011; 52:1535-1540

DOI: 10.2967/jnumed.110.082511

\section{$\mathbf{S}$} tumors, including embryonal and alveolar rhabdomyosarcoma. Most of these entities are characterized by distinct

\footnotetext{
Received Sep. 1, 2010; revision accepted Aug. 1, 2011.

For correspondence or reprints contact: Matthias Weckesser, Klinik und Poliklinik für Nuklearmedizin, Universitätsklinikum Münster, Albert-SchweitzerCampus 1, Gebäude A1, 48149 Münster, Germany.

E-mail: m.weckesser@uni-muenster.de

Published online Sep. 8, 2011.

COPYRIGHT @ 2011 by the Society of Nuclear Medicine, Inc.
}

genetic mutations (e.g., $\mathrm{t}(1 ; 13$ or $2 ; 13)$ in alveolar rhabdomyosarcoma) (1). Soft-tissue sarcomas account for $6 \%-$ $10 \%$ of all childhood malignancies. Rhabdomyosarcoma constitutes approximately $60 \%$ of these.

Although the diagnosis implies the identification of skeletal muscle differentiation, such as myofibrils, cross striations, or desmin and myoD1 (2), rhabdomyosarcoma also occurs at sites where skeletal muscle is not found. Thus, this tumor is more likely derived from primitive mesenchyma with a tendency toward myogenesis than from skeletal muscle (3). The incidence averages between 5 and 7 cases per million children, with a male preponderance.

Although rhabdomyosarcoma is the fourth most common solid tumor in childhood, the annual number of cases is small, necessitating cooperative group trials to better define risk groups and to improve therapy (4-6).

Among risk factors that predict outcome, 6 have come to be universally accepted: tumor size and invasiveness, primary site, grossly complete surgical removal of localized tumor, age of the patient, alveolar or embryonal differentiation, and presence or absence of metastatic involvement (7). On the basis of these criteria, the Cooperative Weichteilsarkom Studie 2002 protocol distinguishes 4 risk groups that vary considerably in prognosis and therapy.

Because of continuous improvement in risk stratification and implementation of an intensive multimodality treatment protocol, event-free survival (EFS) and overall survival (OS) constantly increased from $25 \%$ in 1970 to $70 \% 25$ y later $(8,9)$. Despite this improvement, 5-y EFS for patients with distant metastasis is as low as $25 \%$. Thus, improved diagnostics and consequently improved risk stratification gain even more importance. Tools used for staging rhabdomyosarcoma are clinical examination, laboratory tests, ultrasonography, chest radiography, CT, MRI, and bone scintigraphy (10). In addition, a bone marrow aspiration and lumbar puncture may be necessary.

A growing number of recent studies have focused on the evaluation of PET and PET/CT using ${ }^{18} \mathrm{~F}$-FDG as the tracer (11-13). Soft-tissue sarcomas usually metabolize glucose at an increased rate. Preliminary results for a limited number of cases indicate that staging becomes more accurate when 
${ }^{18} \mathrm{~F}-\mathrm{FDG}$ PET is used as an adjunct to conventional imaging. In other types of sarcomas, ${ }^{18}$ F-FDG PET was predictive of prognosis $(14,15)$. The independent prediction of outcome is a strong indicator of a meaningful diagnostic test.

The aim of this study was to assess the prognostic significance of metabolic tumor activity and stage as determined by ${ }^{18} \mathrm{~F}-\mathrm{FDG}$ PET/CT in patients with rhabdomyosarcoma.

\section{MATERIALS AND METHODS}

\section{Patients}

Forty-one patients with rhabdomyosarcoma who underwent a PET or PET/CT scan for diagnostic purposes were retrospectively identified (17 were female and 24 were male, with an age range of $1-20 \mathrm{y}$ and a mean age $[ \pm \mathrm{SD}]$ of $9.9 \pm 5.8 \mathrm{y}$; examination period, 1998-2008). All patients enrolled in this study had a biopsy-proven rhabdomyosarcoma; other soft-tissue sarcomas were excluded. Patients older than $20 \mathrm{y}$ were not included in the study. The follow-up period ranged from 11 to 141 mo (mean, $49 \pm 38$ mo). All patients received risk-adapted therapy according to the Cooperative Weichteilsarkom Studie 2002 protocol. All procedures were approved by the institutional review board.

\section{Imaging Technique}

Thirty-six PET/CT studies were performed with a hybrid scanner (Biograph Sensation 16; Siemens Medical Solutions) that consists of a full-ring PET tomograph (lutetium oxyorthosilicate crystals) and a 16-detector-row CT component. In 5 patients, attenuation-corrected PET was performed on an ECAT EXACT 921/47 PET scanner (Siemens CTI). All patients were studied after fasting for at least $5 \mathrm{~h}$. Blood glucose levels at the time of ${ }^{18}$ F-FDG application were less than $120 \mathrm{mg} / \mathrm{dL}$. Body-weightadapted activities of ${ }^{18} \mathrm{~F}-\mathrm{FDG}$ ( $4 \mathrm{MBq} / \mathrm{kg}$ of body weight) were injected intravenously $60 \mathrm{~min}$ before the acquisition.

The number of bed positions was adjusted to cover the whole body from head to toes. If patient size exceeded the possible scanning range, the legs were scanned in a second acquisition after the patient had been repositioned. Attenuation was corrected with the segmented low-dose CT data as implemented by the manufacturer. Details concerning ${ }^{18} \mathrm{~F}-\mathrm{FDG}$ PET scans in pediatric patients and techniques have been previously described and were strictly applied in the current study (14).

\section{Imaging Analysis}

All ${ }^{18}$ F-FDG PET/CT images were initially evaluated by a pair of experienced observers - a board-certified radiologist and a board-certified nuclear medicine physician-who were aware of clinical and histopathologic data. The complete evaluation was repeated by a board-certified nuclear medicine physician with 17 $\mathrm{y}$ of experience in PET and $5 \mathrm{y}$ of experience in PET/CT, who was not aware of the staging results. In that analysis, the systematic quantification and classification were performed as outlined below. Consensus on the 2 readings was achieved in all cases after unmasking.

Metabolic activities of the primary tumors were visually classified into 3 groups. Group 1 showed no uptake or only lowintensity uptake (lower than or equal to liver uptake). Group 2 showed intermediate-intensity uptake (higher than liver uptake but considerably lower than brain uptake). Group 3 showed highintensity uptake (not discernible from brain activity using a gray scale typically ranging from 0 to 3.5 times average liver activity). Lymph node and distant metastatic involvement was assessed by PET, and only metabolically active metastases are referred to as N1 or M1 for the purpose of the present study. In a second step, body weight-based maximum standardized uptake value $\left(\mathrm{SUV}_{\max }\right)$ of the primary tumors, lymph nodes, and distant metastases was determined for semiquantitative interpretation. Consecutive circular regions of interest covering the complete tumor diameter were placed on all slices containing tumor or metastases, and the respective $\mathrm{SUV}_{\max }$ of primary tumors, lymph nodes, and metastases was thus determined. $\mathrm{SUV}_{\max }$ was divided by the mean individual SUV of the liver $\left(\mathrm{SUV}_{\text {liver }}\right)$, to account for age- or weight-related differences in whole-body metabolic activity and to avoid systematic errors due to the use of 2 different scanners. Liver activity was measured on a mid-liver axial section as defined on coronal slices. A circular region of interest as large as possible and including liver tissue only was individually drawn, and mean SUV was recorded. In accordance with published data, a cutoff of 6 (SUV $<6$ vs. SUV $\geq 6$ ) was chosen to separate tumors according to their metabolic activities $(16,17)$. A ratio of this $\mathrm{SUV}_{\text {max }}$ and mean $\mathrm{SUV}_{\text {liver }}$ resulted in a cutoff of 4.6.

\section{Patient Data}

Patient data included all information for the clinical assessment of prognosis (Table 1). A variety of other imaging methods, such as MRI ultrasound and contrast-enhanced CT, was used for diagnosis of the primary tumor and for staging. The choice of methods depended, for example, on tumor location and patient age. Thus, a systematic comparison of the methods is not possible and was not the objective of the present analysis. Imaging results, however, were part of the clinical diagnosis.

Furthermore, sensitivities and specificities could not be calculated because only rhabdomyosarcoma patients were included, not controls (patients who had suspected rhabdomyosarcoma but were found to be free of the disease).

\section{Definition of Endpoints}

OS was defined as the time from diagnosis to death from any cause. Event-free survival (EFS) was defined as the time from diagnosis to local recurrence, metastases, progression, or death. Patients who had not experienced an event as defined here were censored at the patients' last contact.

\section{Statistical Analyses}

Statistical analyses were performed using the SPSS 17 software package (IBM). OS and EFS were calculated using the KaplanMeier procedure. For comparison of OS and EFS, a log-rank test was used. Multivariate analyses were accomplished using the Cox survival regression analysis. A stepwise-forward procedure was performed to identify independent prognostic variables. Statistical differences between subgroups with $\log$-rank $P$ less than 0.05 were considered significant.

To refine prediction of outcome, a PET score with 4 groups was introduced. Group 1 consisted of patients with low or moderate activity of the primary tumor. Group 2 consisted of patients with intense primary tumors but both groups without activity in either lymph nodes or metastases. Group 3 consisted of patients with low or moderate activity of the primary tumor but with lymph nodes or metastases. Group 4 consisted of patients with high activity of the primary tumor and with lymph nodes or metastases. 
TABLE 1

Characteristics of the 41 Patients

\begin{tabular}{|c|c|c|}
\hline Characteristic & No. of patients & $\%$ \\
\hline \multicolumn{3}{|l|}{ Sex } \\
\hline Female & 17 & 41 \\
\hline Male & 24 & 59 \\
\hline \multicolumn{3}{|l|}{ Age } \\
\hline$\leq 10 y$ & 21 & 51 \\
\hline$>10 y$ & 20 & 49 \\
\hline \multicolumn{3}{|l|}{ Histology } \\
\hline Embryonal & 17 & 41 \\
\hline Alveolar & 24 & 59 \\
\hline \multicolumn{3}{|c|}{ Site of primary tumor } \\
\hline Orbit & 2 & 5 \\
\hline HN-nPM & 5 & 12 \\
\hline HN-PM & 2 & 5 \\
\hline GU-nBP & 2 & 5 \\
\hline GU-BP & 3 & 7 \\
\hline Extremities & 19 & 46 \\
\hline Other & 8 & 20 \\
\hline \multicolumn{3}{|c|}{ Resection status } \\
\hline Ro & 4 & 10 \\
\hline $\mathrm{R} 1$ & 5 & 12 \\
\hline $\mathrm{R} 2$ & 32 & 78 \\
\hline \multicolumn{3}{|c|}{ Clinical risk group } \\
\hline Group 1 & 0 & 0 \\
\hline Group 2 & 11 & 27 \\
\hline Group 3 & 18 & 44 \\
\hline Group 4 & 12 & 29 \\
\hline
\end{tabular}

$\mathrm{HN}-\mathrm{nPM}=$ head/neck nonparameningeal; HN-PM = head/ neck parameningeal; GU-nBP = genitourinary nonbladder-prostate; GU-BP = genitourinary bladder-prostate; $\mathrm{RO}=$ complete resection; $\mathrm{R} 1=$ microscopic residuals; $\mathrm{R} 2$ = macroscopic residuals.

\section{RESULTS}

\section{Patient Characteristics}

A total of 46 patients were screened for inclusion into this retrospective analysis. Five patients were excluded because they had other types of soft-tissue sarcoma. The remaining 41 tumors (Table 1) showed histology-proven rhabdomyosarcoma. Four patients had resection biopsies of the primary tumors at external institutions before the initial PET/CT.

Altogether, 76 sites with elevated uptake were identified: 41 primary tumor sites, 16 regions with pathologic lymph nodes in 8 patients, and 19 sites with distant metastases in 11 patients (Fig. 1). Seventeen patients had alveolar histology and 24 embryonal. The primary sites comprised the extremities (19 cases), head and neck nonparameningeal sites ( 5 cases), head and neck parameningeal sites ( 2 cases), genitourinary bladder/prostate sites ( 2 cases), genitourinary non-bladder/prostate sites ( 3 cases), the orbit ( 2 cases), and other sites ( 8 cases). None of the patients was stratified to clinical risk group 1, and 11 patients were in the standard group (group 2), with a mean $\mathrm{SUV}_{\max } / \mathrm{SUV}_{\text {liver }}$ of $3.7 \pm 1.9$. Eighteen patients in the risk group 3 had a mean $\mathrm{SUV}_{\max } /$ $\mathrm{SUV}_{\text {liver }}$ of $3.6 \pm 2.3 ; 12$ in group 4 had a mean $\mathrm{SUV}_{\max } /$ $\mathrm{SUV}_{\text {liver }}$ of $5.2 \pm 3.2$.

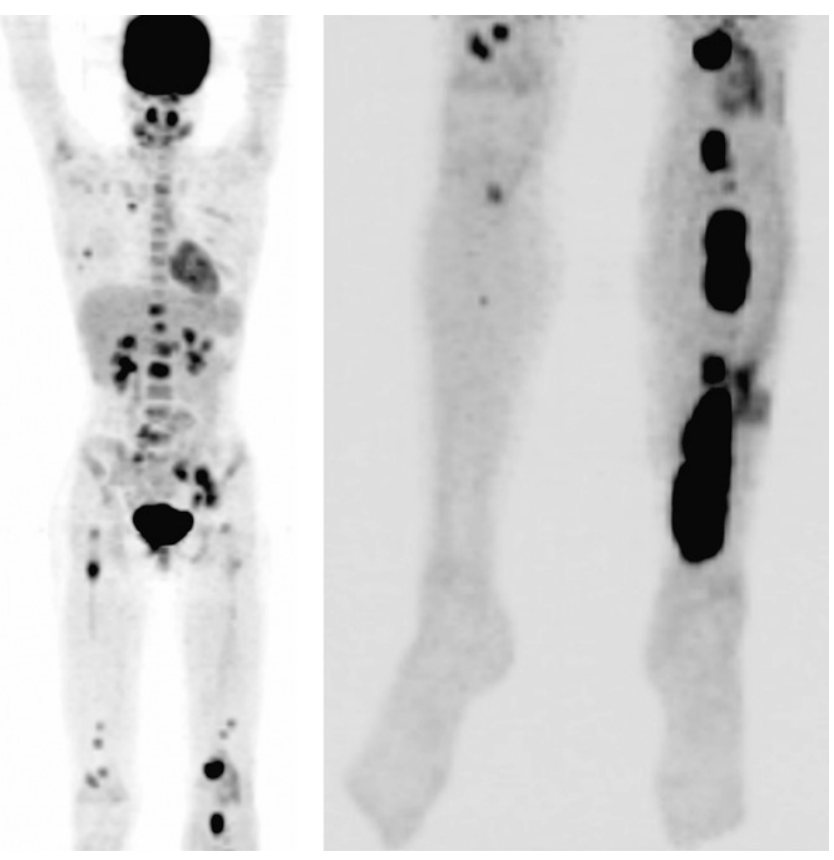

FIGURE 1. A 12-y-old girl with metastatic alveolar rhabdomyosarcoma of the extremity as primarily diagnosed on MRI. Maximumintensity projection of ${ }^{18} \mathrm{~F}$-FDG PET shows multiple lymph node and bone metastases. Patient died 18 mo after initial diagnosis.

The mean follow-up period, including time to death, was $49 \pm 38$ mo. Twelve $(29 \%)$ patients died during this time, and $19(46 \%)$ had an event (local recurrences [5 cases], metastases [8 cases], local recurrences and metastases [5 cases], progression [ 1 case]). There were significant differences in initial $\mathrm{SUV}_{\text {max }} / \mathrm{SUV}_{\text {liver }}$ between patients who died during the observation period and those who survived $(5.8 \pm 2.7$ compared with $3.5 \pm 2.1, P=0.015)$ and patients with or without an event $(5.3 \pm 2.6$ compared with $3.4 \pm 2.1, P=0.026$ ).

MRI of the tumor region was performed in 37 cases. All primary tumors were detected, but affected lymph nodes were missed in 2 cases. Contrast-enhanced CT missed the primary tumor in 4 of 19 cases. Ultrasound of the tumor and the lymphatic drainage depicted lymph nodes in 6 of 9 patients but had a moderate specificity, with 2 falsepositives.

\section{os}

Visually rated metabolic intensity as a discriminator showed significant differences in OS between low-, moderate-, and high-intensity groups $(P=0.007)$ (Fig. 2A). None of the patients with low intensity died $(0 \%)$, but 8 (44\%) patients with high intensity died.

Prediction of OS on the basis of lymph node involvement also showed significant differences $(P=0.016)$ (Fig. 2B). Compared with $16 \%$ of the group without involvement, more than half the patients $(56 \%)$ with metabolically active lymph nodes died.

The presence of metastases decreased OS further (Fig. 2C). According to these data, disparities between patients 


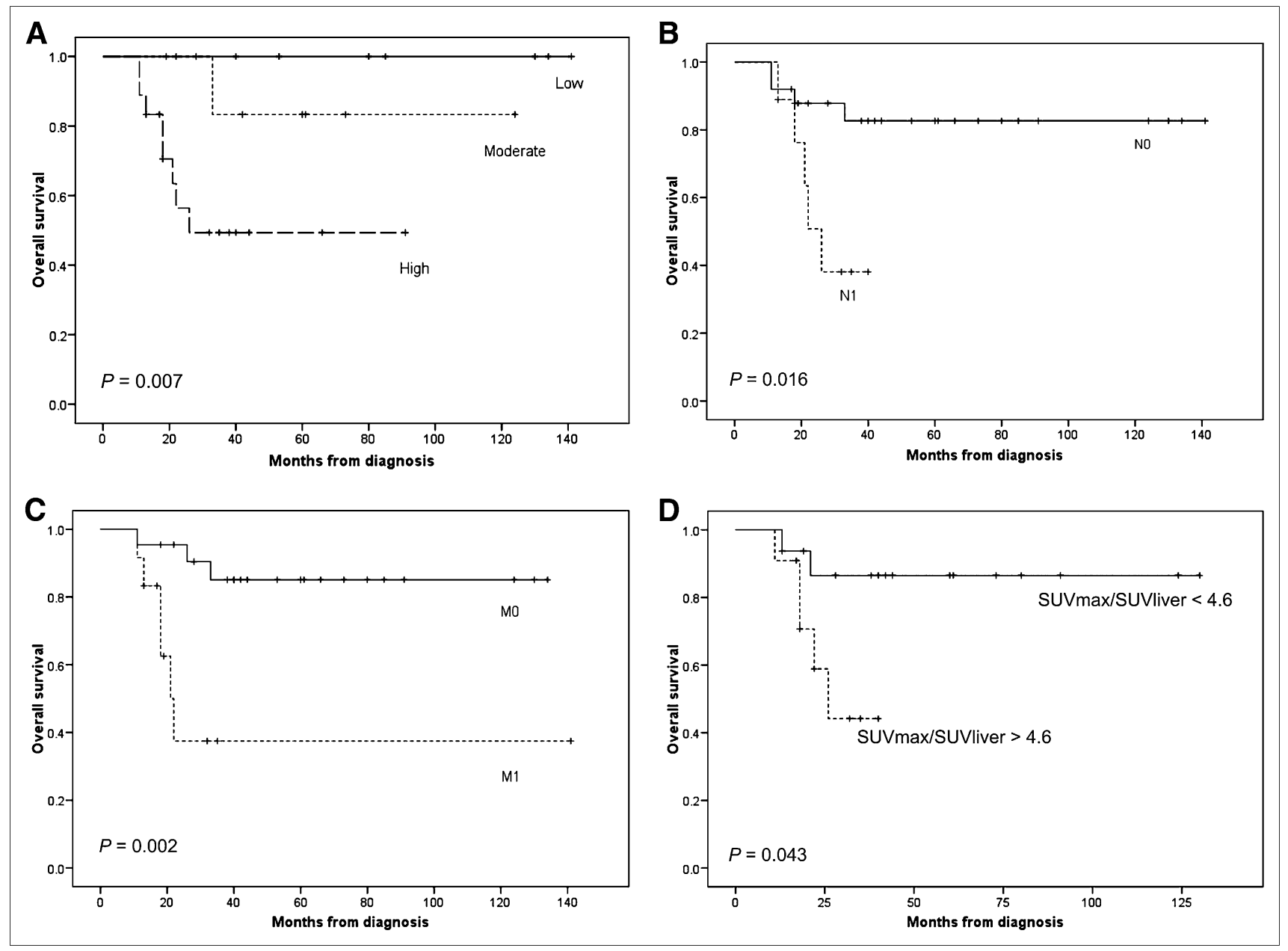

FIGURE 2. Kaplan-Meier OS estimates for primary tumor metabolic intensity (A), lymph node involvement (B), metabolically active metastatic involvement (C), and tumor $\mathrm{SUV}_{\max } / \mathrm{SUV}_{\text {liver }}(\mathrm{D})$.

with and without distant sites were significant $(P=0.002)$. Half of all patients $(50 \%)$ with PET-positive metastases died during follow-up.

A threshold of $\mathrm{SUV}_{\max } / \mathrm{SUV}_{\text {liver }}$ of 4.6 for the primary tumor discriminated between 2 groups with significant differences in OS survival (log-rank $P=0.002)$. This cutoff was confirmed by ROC analysis.

The number of patients with M1 (11 cases) or N1 (8 cases), and especially the fraction with a metabolic activity above the threshold of 4.6 ( 2 patients for M1, both of whom died), were too small to assess the effect of intensity of metastases on survival.

\section{EFS}

In a second step, Kaplan-Meier curves for EFS were plotted. All results are depicted in Table 2 and are comparable to the findings of OS. Primary tumor intensity as well as lymph node and distant metastatic involvement showed significant differences between groups (primary tumor low vs. moderate vs. high, $P=0.005$; N0 vs. N1, $P=0.008$; M0 vs. M1, $P=0.01$ ). The semiquantitative evaluation of the primary tumor failed to attain statistical significance for
EFS $\left(\mathrm{SUV}_{\max } / \mathrm{SUV}_{\text {liver }}<4.6\right.$ compared with $\mathrm{SUV}_{\max } /$ $\mathrm{SUV}_{\text {liver }} \geq 4.6, P=0.139$ ).

\section{PET Score}

Kaplan-Meier analysis revealed significant differences in OS among the 4 groups (Fig. 3, $P=0.002$ ). Whereas none of group $\mathrm{T}_{\text {low }}+$ moderate, $\mathrm{N} / \mathrm{M} 0$, died $(0 \%)$, more than half the patients in group $\mathrm{T}_{\text {intense }}, \mathrm{N} / \mathrm{M} 1$, died of disease $(55 \%)$. Comparable results were calculated for EFS (Table 2; $P=$ $0.023)$.

\section{Multivariate Analysis for Prognostic Factors and Tumor Intensity}

The prediction of survival by primary tumor intensity was assessed using Cox regression analysis. Accepted prognostic factors were included. A highly significant effect of patient age was found $(P=0.001)$. In contrast, primary tumor intensity failed to attain statistical significance $(P=0.081)$. This was also true for the factors histology, tumor size, primary tumor location risk, and postsurgical status, which showed lower $P$ values than tumor intensity. The presence of lymph node and hematogenic metastatic 
TABLE 2

Results of Kaplan-Meier EFS Estimates for Different Primary Tumor Metabolic Activities and Presence of Nodal or Hematogenic Metastases on PET

\begin{tabular}{|c|c|c|c|}
\hline Group & $\begin{array}{c}\text { No. of } \\
\text { endpoints }\end{array}$ & $\begin{array}{c}\% \text { of } \\
\text { patients }\end{array}$ & $P$ \\
\hline $\mathrm{PT}_{\text {low }}$ & 1 & 13 & 0.005 \\
\hline $\mathrm{PT}_{\text {moderate }}$ & 2 & 25 & \\
\hline $\mathrm{PT}_{\text {high }}$ & 10 & 63 & \\
\hline No & 7 & 29 & 0.008 \\
\hline N1 & 6 & 75 & \\
\hline MO & 5 & 24 & 0.01 \\
\hline M1 & 8 & 73 & \\
\hline $\mathrm{PT} \mathrm{SUV}_{\max } /$ SUV $_{\text {liver }}<4.6$ & 4 & 27 & 0.139 \\
\hline $\mathrm{PT}_{\mathrm{SUV}_{\max } / \mathrm{SUV}_{\text {liver }}} \geq 4.6$ & 6 & 55 & \\
\hline $\mathrm{PT}_{\text {low }}+$ moderate $; \mathrm{N} / \mathrm{M} 0$ & 1 & 9 & 0.023 \\
\hline $\mathrm{PT}_{\text {intense }} ; \mathrm{N} / \mathrm{M} 0$ & 2 & 33 & \\
\hline $\mathrm{PT}_{\text {low }}+$ moderate $; \mathrm{N} / \mathrm{M} 1$ & 3 & 75 & \\
\hline $\mathrm{PT}_{\text {intense }} ; \mathrm{N} / \mathrm{M} 1$ & 7 & 70 & \\
\hline $\mathrm{PT}=$ primary tumor. & & & \\
\hline
\end{tabular}

involvement attained significance. However, retrospectively, it was hard to determine in how many cases the metastatic foci would have been missed or misinterpreted by conventional imaging. A masked comparative imaging study to assess how many sites of metastasis would have been found without PET and how the additional sites might improve the prediction of prognosis was not part of the present study.

\section{DISCUSSION}

The diagnosis, treatment, and reevaluation of soft-tissue sarcoma are complex and are primarily the task of specialized centers. Conventional imaging, such as CT, ultrasound, or MRI, is well established. Initial results of PET and PET/CT in the diagnosis and risk stratification of patients with rhabdomyosarcoma are promising. PET can

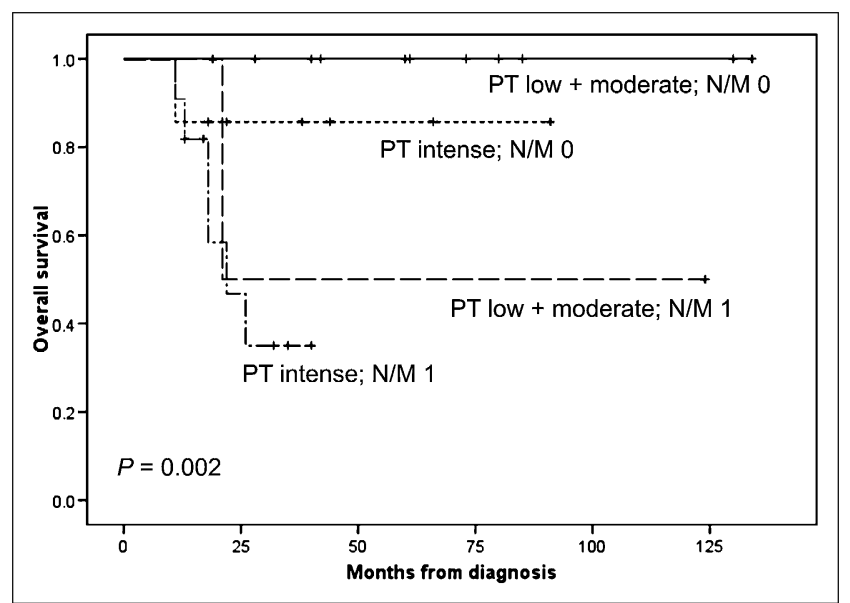

FIGURE 3. Kaplan-Meier OS estimates for suggested PET score. be used to assess the primary tumor and to improve staging $(18,19,20)$. Results in other sarcomas such as osteosarcoma, chondrosarcoma, and Ewing tumors indicate that PET/CT is predictive of outcome $(14,15,17,21)$. On the other hand, the sarcoma population represents a heterogeneous group and each type of tumor has to be studied separately. In relation to this need, the aim of the present analysis was to assess the potential of ${ }^{18} \mathrm{~F}-\mathrm{FDG}$ PET/CT for prediction of OS and EFS in patients with rhabdomyosarcoma.

As demonstrated in Figure 2, a correlation between low, moderate, or high intensity of the primary tumor and OS was observed. In the high-intensity group, $44 \%$ of patients died, whereas no patient of the low-intensity group died during the follow-up period. Kaplan-Meier curves for PET-positive lymph node and distant site participation revealed significant differences too, with patients showing a worse outcome when these locations were affected. Furthermore, the ratio $\mathrm{SUV}_{\max } / \mathrm{SUV}_{\text {liver }}$ with the cutoff 4.6 in the present study appears to be a discriminator for OS as well. Similar results were found in EFS in relation to tumor intensity, lymph node metastasis, and distant site metastasis.

The visual intensity classification used in the present study was based on a simple comparison of tumor activity with liver and brain activity. This comparison depends both on the examiner's experience and on a continuous assessment. However, $\mathrm{SUV}_{\text {max }} / \mathrm{SUV}_{\text {liver }}$ quantification is cutoffdependent. Both methods have advantages and drawbacks but both provide information on prognosis.

In a second step, all patients were divided into 4 groups (Fig. 3) to include all the PET information and to obtain more detailed information on prognosis. The subgroups showed significant differences in survival indicating that primary tumor intensity and presence of PET-positive metastases yield complementary information that may refine stratification in future trials.

In a third step, a multivariate analysis was performed on all known rhabdomyosarcoma prognostic factors and primary tumor intensity or $\mathrm{SUV}_{\text {max }} / \mathrm{SUV}_{\text {liver }}$ This analysis lacked statistical significance to prove that primary tumor intensity or $\mathrm{SUV}_{\text {max }} / \mathrm{SUV}_{\text {liver }}$ is an independent predictor of OS and EFS, probably because of the strong effects of patient age and the limited size of the patient sample. This assumption is supported by the other accepted prognostic parameters that lacked significance more clearly in the present analysis.

Rhabdomyosarcoma of childhood is a rare disease. Thus, a drawback of the present study was its limited number of patients. By enhancing the number of enrolled subjects, it may be possible to obtain more detailed information and to develop advanced predictive models.

The present cohort exhibits a center bias such that a relatively large number of patients with alveolar histology was included (59\% in contrast to $20 \%$ in the literature) (1). We thus discovered a higher subcategory relating to the location of the primary tumor. More precisely, $44 \%$ of our collective had an extremity rhabdomyosarcoma (19\% 
in the literature) (22). Both an alveolar histology and a primary rhabdomyosarcoma in an extremity show a worse outcome and may lead to a selection bias.

The present study was not designed as a comparative imaging study. In general, MRI is probably the best method to evaluate tumor location and involvement of anatomic structures in the tumor vicinity and is thus a prerequisite for surgical therapy.

The present image analyses support the finding that PET is not the most effective tool to visualize lung metastases (23), most likely because of the limited spatial resolution of PET and the blurring induced by respiratory motion. The accuracy of CT is unsurpassed for the depiction of lung metastases, especially in children, because the likelihood of unspecific nodules is lower in children than in adults. Using hybrid scanners, the CT part of the study can be analyzed for lung metastases.

\section{CONCLUSION}

${ }^{18} \mathrm{~F}$-FDG PET/CT is a valuable tool for initial staging of rhabdomyosarcoma patients, seems to be an additional predictor of outcome, and may contribute to further improvement of survival by refined risk-adapted therapy (22). The proposed PET score may be helpful as a new predictive parameter but still needs validation in a larger cohort.

\section{DISCLOSURE STATEMENT}

The costs of publication of this article were defrayed in part by the payment of page charges. Therefore, and solely to indicate this fact, this article is hereby marked "advertisement" in accordance with 18 USC section 1734.

\section{ACKNOWLEDGMENT}

No potential conflict of interest relevant to this article was reported.

\section{REFERENCES}

1. Dagher R, Helman L. Rhabdomyosarcoma: an overview. Oncologist. 1999;4: 34-44.

2. Qualman SJ, Coffin CM, Newton WA, et al. Intergroup Rhabdomyosarcoma Study: update for pathologists. Pediatr Dev Pathol. 1998;1:550-561.

3. Parham DM. Pathologic classification of rhabdomyosarcomas and correlations with molecular studies. Mod Pathol. 2001;14:506-514.

4. Pappo AS, Anderson JR, Crist WM, et al. Survival after relapse in children and adolescents with rhabdomyosarcoma: a report from the Intergroup Rhabdomyosarcoma Study Group. J Clin Oncol. 1999;17:3487-3493.
5. Crist WM, Anderson JR, Meza JL, et al. Intergroup rhabdomyosarcoma studyIV: results for patients with nonmetastatic disease. J Clin Oncol. 2001;19:30913102 .

6. Raney RB, Maurer HM, Anderson JR, et al. The Intergroup Rhabdomyosarcoma Study Group (IRSG): major lessons from the IRS-I through IRS-IV studies as background for the current IRS-V treatment protocols. Sarcoma. 2001;5: 9-15.

7. Raney RB, Anderson JR, Barr FG, et al. Rhabdomyosarcoma and undifferentiated sarcoma in the first two decades of life: a selective review of intergroup rhabdomyosarcoma study group experience and rationale for Intergroup Rhabdomyosarcoma Study V. J Pediatr Hematol Oncol. 2001;23:215-220.

8. Dantonello TM, Int-Veen C, Harms D, et al. Cooperative trial CWS-91 for localized soft tissue sarcoma in children, adolescents, and young adults. J Clin Oncol. 2009;27:1446-1455.

9. Brecht IB, Treuner J. Soft tissue sarcoma in children and adolescents: experiences of the cooperative Soft Tissue Sarcoma Group Studies (CWS-81 - 96). Handchir Mikrochir Plast Chir. 2004;36:275-281.

10. Van Rijn RR, Wilde JC, Bras J, Oldenburger F, McHugh KM, Merks JH. Imaging findings in noncraniofacial childhood rhabdomyosarcoma. Pediatr Radiol. 2008;38:617-634.

11. Klem ML, Grewal RK, Wexler LH, Schoder H, Meyers PA, Wolden SL. PET for staging in rhabdomyosarcoma: an evaluation of PET as an adjunct to current staging tools. J Pediatr Hematol Oncol. 2007;29:9-14.

12. Lucas JD, O'Doherty MJ, Cronin BF, et al. Prospective evaluation of soft tissue masses and sarcomas using fluorodeoxyglucose positron emission tomography. Br J Surg. 1999;86:550-556.

13. Folpe AL, Lyles RH, Sprouse JT, Conrad EU III, Eary JF. (F-18) fluorodeoxyglucose positron emission tomography as a predictor of pathologic grade and other prognostic variables in bone and soft tissue sarcoma. Clin Cancer Res. 2000;6:1279-1287.

14. Franzius C, Bielack S, Flege S, Sciuk J, Jurgens H, Schober O. Prognostic significance of ${ }^{18} \mathrm{~F}$-FDG and ${ }^{99 \mathrm{~m}} \mathrm{Tc}$-methylene diphosphonate uptake in primary osteosarcoma. J Nucl Med. 2002;43:1012-1017.

15. Brenner W, Conrad EU, Eary JF. FDG PET imaging for grading and prediction of outcome in chondrosarcoma patients. Eur J Nucl Med Mol Imaging. 2004;31: 189-195.

16. Schuetze SM, Rubin BP, Vernon C, et al. Use of positron emission tomography in localized extremity soft tissue sarcoma treated with neoadjuvant chemotherapy. Cancer. 2005;103:339-348.

17. Eary JF, O'Sullivan F, Powitan Y, et al. Sarcoma tumor FDG uptake measured by PET and patient outcome: a retrospective analysis. Eur J Nucl Med Mol Imaging. 2002;29:1149-1154.

18. Ben Arush MW, Bar Shalom R, Postovsky S, et al. Assessing the use of FDGPET in the detection of regional and metastatic nodes in alveolar rhabdomyosarcoma of extremities. J Pediatr Hematol Oncol. 2006;28:440-445.

19. McCarville MB, Christie R, Daw NC, Spunt SL, Kaste SC. PET/CT in the evaluation of childhood sarcomas. AJR. 2005;184:1293-1304.

20. Tateishi U, Hosono A, Makimoto A, et al. Comparative study of FDG PET/CT and conventional imaging in the staging of rhabdomyosarcoma. Ann Nucl Med. 2009;23:155-161.

21. Gerth HU, Juergens KU, Dirksen U, Gerss J, Schober O, Franzius C. Significant benefit of multimodal imaging: PET/CT compared with PET alone in staging and follow-up of patients with Ewing tumors. J Nucl Med. 2007;48:1932-1939.

22. Dantonello TM, Int-Veen C, Winkler P, et al. Initial patient characteristics can predict pattern and risk of relapse in localized rhabdomyosarcoma. J Clin Oncol. 2008;26:406-413.

23. Völker T, Denecke T, Steffen I, et al. Positron emission tomography for staging of pediatric sarcoma patients: results of a prospective multicenter trial. J Clin Oncol. 2007;25:5435-5441. 